\section{PWE-107 NOVEL PROGNOSTIC TARGETS IDENTIFIED USING INTEGRATED ANALYSIS OF COPY NUMBER AND EXPRESSION DATA IN OESOPHAGEAL ADENOCARCINOMA}

\author{
doi:10.1136/gut.2011.239301.370
}

X Y Goh, ${ }^{1, *}$ M A Alvi, ${ }^{1} \mathrm{~J}$ Rees, ${ }^{1}$ S F Chin, ${ }^{2} \mathrm{~J}$ Weaver, ${ }^{1} \mathrm{C}$ Caldas, ${ }^{2} \mathrm{R}$ Fitzgerald ${ }^{1} \mathrm{MRC}$ Cancer Cell Unit, Hutchison-MRC Research Centre, Cambridge, UK; ${ }^{2}$ Cancer Research UK Cambridge Research Institute, Cambridge, UK

Introduction The incidence of oesophageal adenocarcinoma $(\mathrm{OAC})$ has increased sixfold in the west over the last 30 years, with a dismal survival rate of less than $20 \%$. Understanding the genomic aberrations and biology of this cancer may enhance disease interventions. We aim to identify novel molecular targets with clinical relevance using an integrated approach in analysing genomewide data of $\mathrm{OAC}$, and to correlate reduced expression of molecular targets with promoter hypermethylation in OAC.

Methods $30 \mathrm{k}$ oligonucleotide array comparative genomic hybridisation (CGH) and gene expression microarray profiling were performed on $56 \mathrm{OAC}$ resection samples with long-term clinical follow-up data to identify regions of copy number changes leading to differential expression levels. Fluorescence in situ hybridisation (FISH) assays were carried out using bacterial artificial chromosome probes on touch-imprinted slides of frozen tumour samples to confirm their amplification status. Immunohistochemistry (IHC) assays on tissue microarrays (TMAs) on the same cohort $(n=56)$ and on an independent cohort of OAC samples $(n=371)$ were used for subsequent validation at the protein level. Kaplan-Meier survival analyses were performed based on IHC data to determine prognostic significance of novel molecular targets. An Illumina Infinium Array was used to investigate the methylation status of 24 independent $\mathrm{OAC}$ tumours, compared with 24 premalignant Barrett's Oesophagus (BE) samples.

Results Integrated array CGH data with matched gene expression microarray data highlighted 4 genes with copy number gains and increased expression: EGFR, WT1, NEIL2 and MTMR9. FISH assays confirmed amplifications of these genes ( $>6$ copies/centromere) in $11 \%$ of OAC samples and IHC assays ( $\mathrm{n}=371$ independent cases) demonstrated protein over-expression: EGFR (10\%), WT1 (20\%), NEIL2 (14\%) and MTMR9 (25\%), corroborating findings from the integrated analysis. These targets individually had prognostic significance $(p<0.060)$. Interestingly, reduced expressions of WT1 $(\mathrm{p}=0.037)$ and NEIL2 $(\mathrm{p}=0.051)$ were associated with poorer prognosis in OAC. In order to investigate the mechanism of reduced expression of these targets which might have contributed to a worse prognosis, methylation array data was utilised from an independent dataset. This showed increased methylation of WT1 in OAC (63\%) compared to BE $(55 \%$; t test $\mathrm{p}=0.028)$ and normal controls $(50 \%)$, but no difference in methylation status of NEIL2 between disease states.

Conclusion Integration of array CGH and gene expression data identified novel genes not previously associated with $\mathrm{OAC}$ with prognostic potential that require further study.

Competing interests None.

Keywords amplification, oesophageal adenocarcinoma, overexpression, prognosis. 